\title{
Sex-related impact on clinical outcomes of patients treated with drug-eluting stents according to clinical presentation: Patient-level pooled analysis from the GRAND-DES registry
}

Eun-Seok Shin ${ }^{1}$, Eun Jung Jun ${ }^{1}$, Jung-Kyu Han², Min Gyu Kong², Jeehoon Kang ${ }^{2}$, Chengbin Zheng ${ }^{2}$, Scot Garg ${ }^{3}$, Young Jin Choi ${ }^{4}$, Jang-Whan Bae ${ }^{5}$, Kook-Jin Chun ${ }^{6}$, Doo-Il Kim ${ }^{7}$, Seung-Woon Rha ${ }^{8}$, Sung Yun Lee ${ }^{9}$, Jay Young Rhew ${ }^{10}$, Seong-Ill Woo ${ }^{11}$, Han Cheol Lee ${ }^{12}$, Jin-Ok Jeong ${ }^{13}$, Han-Mo Yang ${ }^{2}$, Kyung Woo Park ${ }^{2}$, Hyun-Jae Kang ${ }^{2}$, Bon-Kwon $\mathrm{Koo}^{2}$, In-Ho Chae ${ }^{14}$, Hyo-Soo Kim ${ }^{2}$

${ }^{1}$ Department of Cardiology, Ulsan Hospital, Ulsan, Korea; Department of Cardiology, Ulsan University Hospital, University of Ulsan College of Medicine, Ulsan, Korea; ${ }^{2}$ Cardiovascular Center, Seoul National

University Hospital, Seoul, Republic of Korea; ${ }^{3}$ East Lancashire Hospitals NHS Trust, Blackburn, Lancashire, United Kingdom; ${ }^{4}$ Division of Cardiology, Department of Internal Medicine, Sejong General

Hospital, Bucheon, Republic of Korea; ${ }^{5}$ Division of Cardiology, Department of Internal Medicine, Chungbuk National University College of Medicine, Cheongju, Republic of Korea; ${ }^{6}$ Division of Cardiology, Department of Internal Medicine, Pusan National University Yangsan Hospital, Yangsan, Republic of Korea; ${ }^{7}$ Department of Internal Medicine, Haeundae Paik Hospital, Inje University College of Medicine, Busan, Republic of Korea; ${ }^{8}$ Cardiovascular Center, Korea University Guro Hospital, Seoul, Republic of Korea; ${ }^{9}$ Division of Cardiology, Department of Internal Medicine, Inje University Ilsan Paik Hospital, Goyang, Republic of Korea; ${ }^{10}$ Department of Internal Medicine and Cardiovascular Center, Presbyterian Medical Center, Jeonju, Republic of Korea; ${ }^{11}$ Division of Cardiology, Department of Internal Medicine, Inha University Hospital, Incheon, Republic of Korea; ${ }^{12}$ Division of Cardiology, Department of Internal Medicine, Pusan National University Hospital, Busan, Republic of Korea; ${ }^{13}$ Division of Cardiology, Department of Internal Medicine, Chungnam National University School of Medicine, Daejon, Republic of Korea; ${ }^{14}$ Cardiovascular Center, Seoul National University Bundang Hospital,

Seongnam-si, Republic of Korea

\begin{abstract}
Background: The contribution of sex and initial clinical presentation to the long-term outcomes in patients undergoing percutaneous coronary intervention (PCI) is still debated.

Methods: Individual patient data from 5 Korean-multicenter drug-eluting stent (DES) registries (The GRAND-DES) were pooled. A total of 17,286 patients completed 3-year follow-up (5216 women and 12,070 men). The median follow-up duration was 1125 days (interquartile range 1097-1140 days), and the primary endpoint was cardiac death at 3 years.

Results: The clinical indication for PCI was stable angina pectoris (SAP) in 36.8\%, unstable angina pectoris (UAP) or non-ST-segment elevation myocardial infarction (NSTEMI) in 47.4\%, and
\end{abstract}

Address for correspondence: Hyo-Soo Kim, MD, PhD, FAHA, Cardiovascular Center, Seoul National University Hospital, 101 Daehak-ro, Chongno-gu, Seoul 03080, Republic of Korea, tel: 82-2-2072-2226, fax: 82-2-766-8904, e-mail: usahyosoo@gmail.com

Received: 17.04.2020 Accepted: 8.11.2020 Early publication date: 20.01.2021

This article is available in open access under Creative Common Attribution-Non-Commercial-No Derivatives 4.0 International (CC BY-NC-ND 4.0) license, allowing to download articles and share them with others as long as they credit the authors and the publisher, but without permission to change them in any way or use them commercially. 
ST-segment elevation myocardial (STEMI) in 15.8\%. In all groups, women were older and had a higher proportion of hypertension and diabetes mellitus compared with men. Women presenting with STEMI were older than women with SAP, with the opposite seen in men. There was no sex difference in cardiac death for SAP or UAP/NSTEMI. In STEMI patients, the incidence of cardiac death (7.9\% vs. 4.4\%, $p=0.001)$, all-cause mortality $(11.1 \%$ vs. $6.9 \%, p=0.001)$, and minor bleeding $(2.2 \%$ vs. $1.2 \%$, $p=0.043$ ) was significantly higher in women. After multivariable adjustment, cardiac death was lower in women for UAP/NSTEMI (HR 0.69, 95\% CI 0.53-0.89, $p=0.005)$, while it was similar for STEMI (HR 0.97, 95\% CI 0.65-1.44, $p=0.884$ ).

Conclusions: There was no sex difference in cardiac death after PCI with DES for SAP and UAP/ /NSTEMI patients. In STEMI patients, women had worse outcomes compared with men; however, after the adjustment of confounders, female sex was not an independent predictor of mortality. (Cardiol J 2023; 30, 1: 105-116)

Key words: coronary artery disease, percutaneous coronary intervention, drug-eluting stent, sex difference, myocardial infarction

\section{Introduction}

Although the mortality from coronary artery disease (CAD) has decreased due to improved prevention and treatment strategies, it remains one of the leading causes of morbidity and mortality in men and women worldwide. Previous studies have suggested that, compared with men, women were at higher risk of worse outcomes following percutaneous coronary intervention (PCI), with differences in risk factors thought to be the cause [1]. Women undergoing PCI tend to be older with a significantly higher prevalence of diabetes and hypertension. Men are more likely to be smokers, and have multivessel disease and higher rates of prior myocardial infarction (MI). After adjustment of confounding factors, there have been conflicting results. Recent studies have suggested that gender differences have diminished, possibly through the evolution of PCI-related devices and evidence-based medical therapy [2, 3]. To date, there are only a few studies comparing long-term clinical outcomes following PCI with drug-eluting stents (DES) between men and women in different clinical presentations. Therefore, we aimed to examine gender differences in long-term clinical outcomes according to clinical presentation following PCI with DES.

\section{Methods}

The GRAND-DES registry is a patient-level pooled registry consisting of 17,286 patients from 5 separate clinical trials: the Efficacy of Xience/Promus versus Cypher in rEducing Late Loss after stENTing (EXCELLENT) registry (NCT00698607), the Registry to Evaluate the
Efficacy of Zotarolimus-Eluting Stent (RESOLUTE-KOREA) (NCT00960908), the Efficacy and Safety of Xience in Coronary artEry Disease aLLcomers After stenting Using the PRIME Platform (EXCELLENT-PRIME) registry (NCT01605721), the Harmonizing Optimal Strategy for Treatment of coronary artery disease using a BIOLIMUS A9-eluting stent (HOST-BIOLIMUS-3000-Korea) registry, and the Harmonizing Optimal Strategy for Treatment of coronary artery disease using a RESOLute INTEgrity (HOST-RESOLINTE) registry (Fig. 1). The individual trial designs and primary results have been published previously [4-6]. Each trial included in this analysis complied with the Declaration of Helsinki, and the study protocols were approved by the Institutional Review Board at each participating center. All patients provided written informed consent for participation in each study.

The primary endpoint of this study was cardiac death at 3 years. All-cause death, MI, stroke, clinically driven target lesion revascularization (TLR), clinically driven target vessel revascularization (TVR), and definite or probable stent thrombosis (ST) were defined according to the Academic Research Consortium (ARC) definitions, whilst bleeding rates were reported according to the Thrombolysis in Myocardial Infarction bleeding criteria [7]. Decreases in hemoglobin levels of $5 \mathrm{~g} / \mathrm{dL}$ or more occurring during hospitalization or intracranial hemorrhage were classified as major bleeding. Scheduled yearly surveillance was performed routinely after PCI by trained personnel. If death occurred, details were obtained through review of local and external medical records, death certification, telephone contact with patients' family, and external providers. Experienced data 


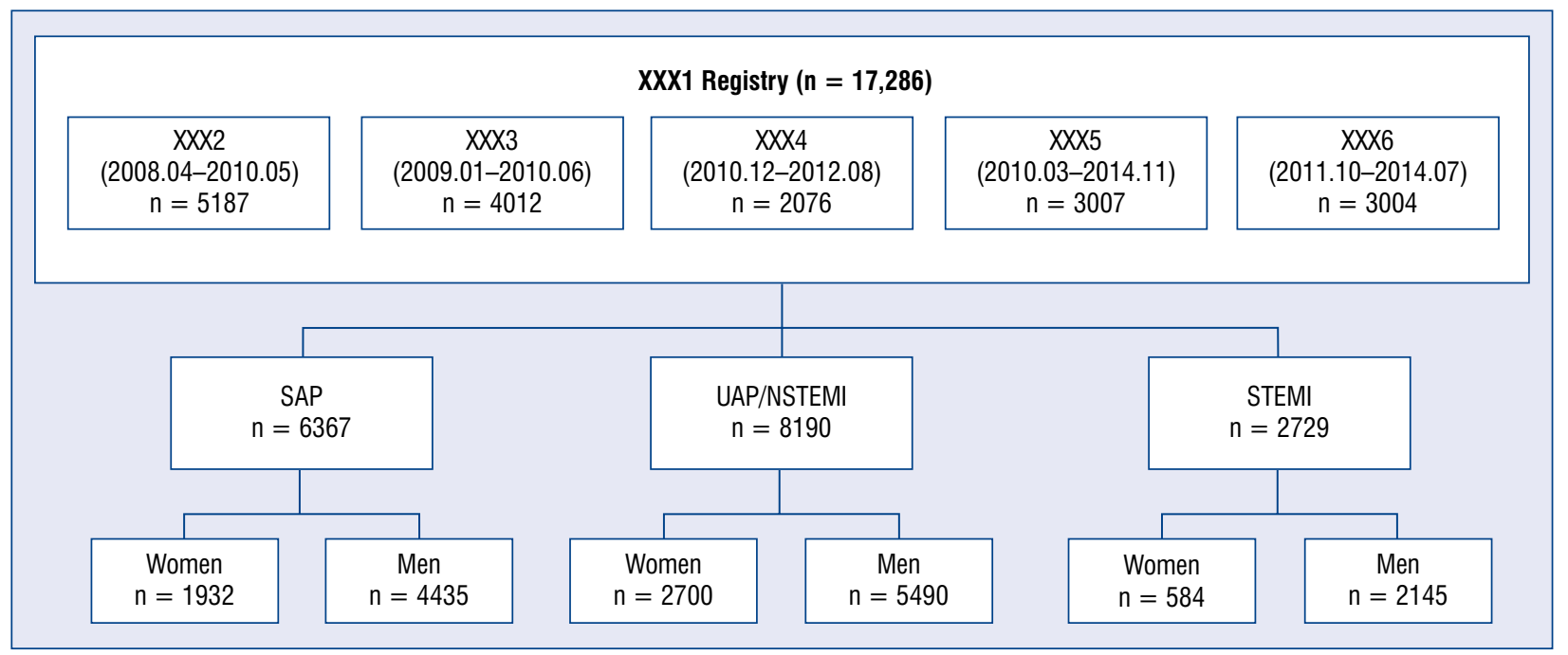

Figure 1. Study flow chart; NSTEMI — non-ST elevation myocardial infarction; SAP — stable angina pectoris; STEMI — ST-segment elevation myocardial infarction; UAP — unstable angina pectoris.

analysts recorded the details of each death and performed initial classification.

\section{Statistical analysis}

We compared the baseline characteristics, treatment, and clinical outcomes between women and men. Baseline characteristics were reported as percentages for categorical variables and means with standard deviation (SD) for continuous variables. Comparisons between groups were made either by Pearson's $\chi^{2}$ test for baseline categorical variables or Student's t-test for continuous variables, as appropriate. In the comparison of clinical outcomes among groups, the cumulative incidences of the primary and secondary endpoints were estimated using the Kaplan-Meier method, and the curves were compared using the log-rank test. Cox proportional hazard survival models were applied for risk comparison between groups. For multivariable analysis, the covariates included in the analysis were age, clinical risk factors, and angiographic and procedural risk factors. To assess the effect of confounders different models were compared. Model 1 was the adjusted model with age only. Model 2 was adjusted with clinical risk factors such as hypertension, diabetes, dyslipidemia, smoking, prior MI, prior PCI, prior congestive heart failure, and prior peripheral vascular disease in addition to model 1. Model 3 was adjusted with angiographic risk factors such as multivessel disease, left main disease, bifurcation, long lesion, number of stents, stent diameter, stent length, and glycoprotein IIb/ /IIIa inhibitor and intravascular ultrasound use, in addition to model 2 . The independent predictors of primary outcome were also obtained by multiple Cox regression analysis. All analyses were performed using $\mathrm{R}$ statistical software 3.6.1 ( $\mathrm{R}$ foundation for Statistical Computing, Vienna, Austria). All $\mathrm{p}$ values were two sided, and a value of $<0.05$ was considered statistically significant.

\section{Results}

A total of 17,286 patients from 5 Korean multicenter DES registries were enrolled in the current GRAND-DES registry. Of these patients, 997 died at 3 -year follow-up. Figure 1 shows the flow of patients entered into the study. The study cohort was made up of $5216(30.2 \%)$ women and $12,070(69.8 \%)$ men, with the clinical indication for PCI stable angina pectoris (SAP) in $36.8 \%$, unstable angina pectoris (UAP) or non-ST-segment elevation myocardial infarction (NSTEMI) in $47.4 \%$, and ST-segment elevation myocardial infarction (STEMI) in $15.8 \%$.

Table 1 shows the clinical characteristics of the study population according to sex and clinical presentation. In all three groups, women were older and had a higher proportion of hypertension and diabetes and a lower rate of current smoking. Women presenting with SAP or UAP/NSTEMI were less likely to have had a previous MI or PCI and more likely to have a history of congestive heart failure, while no such differences occurred in the STEMI group. In terms of medication at discharge, calcium channel blockers were used more frequently in women in all three groups. 


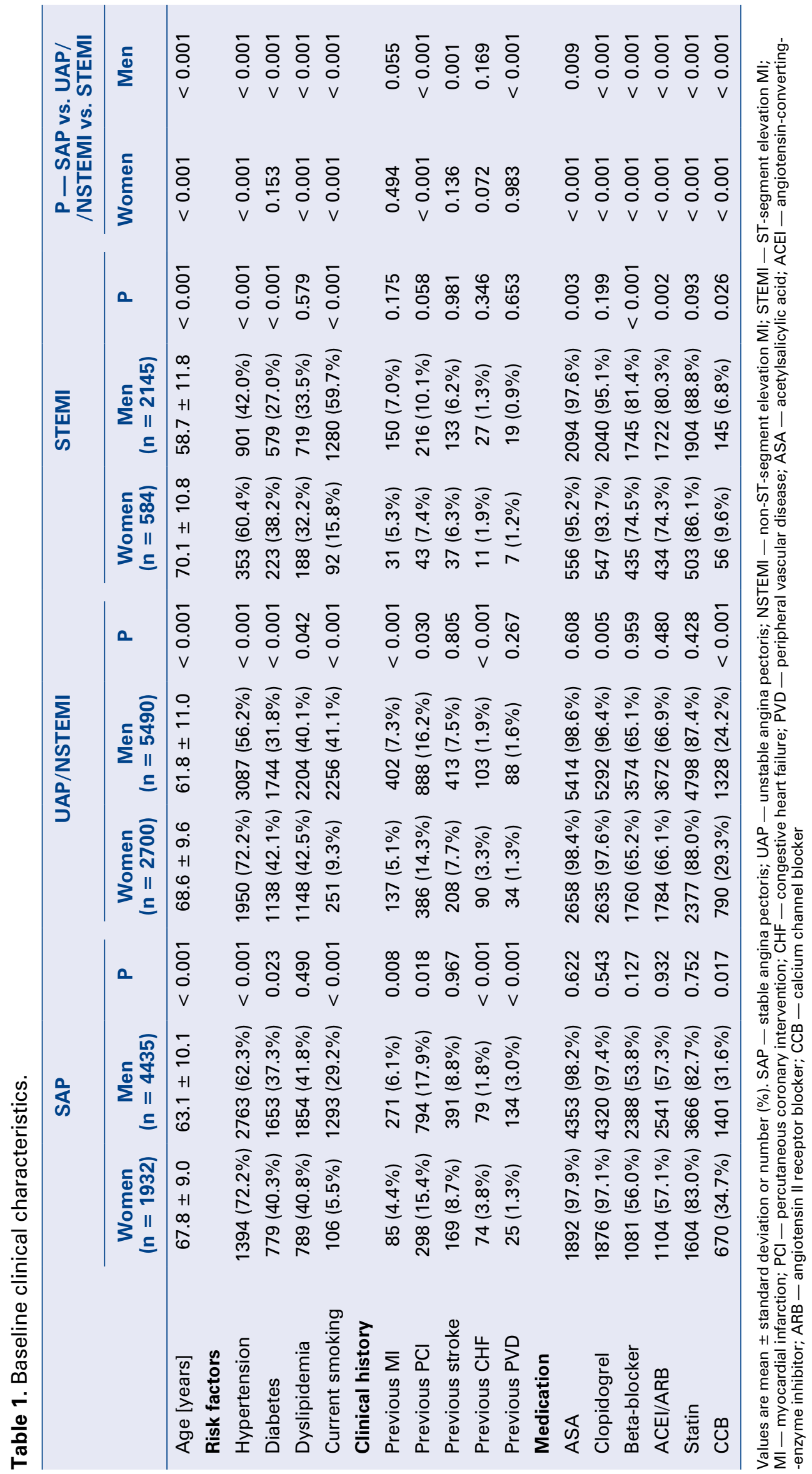




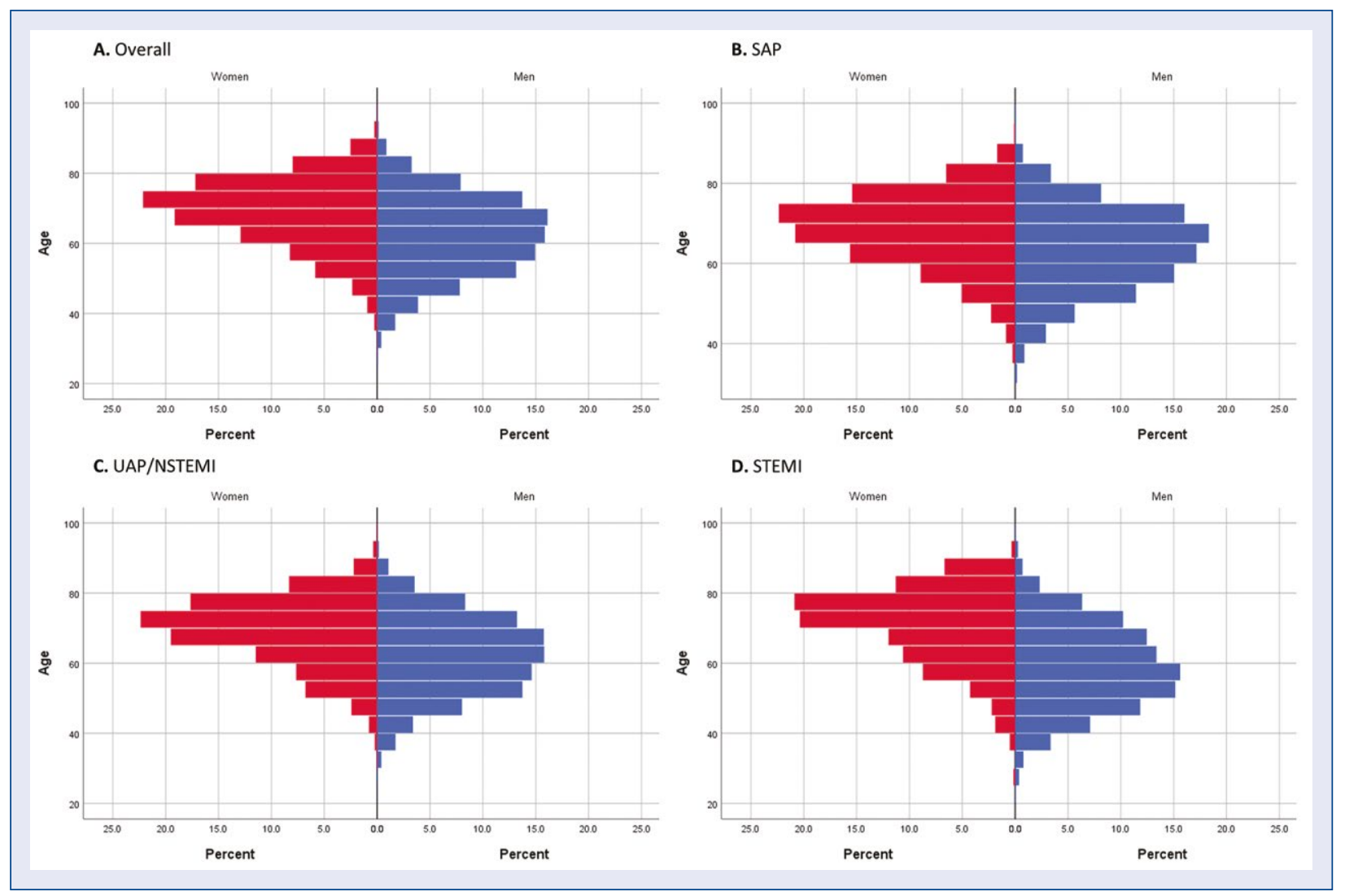

Figure 2. Population of age according to clinical presentation; A. Overall; B. Stable angina pectoris (SAP); C. Unstable angina pectoris/non-ST-segment elevation myocardial infarction (UAP/NSTEMI); D. ST-segment elevation myocardial infarction (STEMI).

Other medications were optimally prescribed, with respective rates of acetylsalicylic acid, clopidogrel, and statin use $>95 \%,>93 \%$, and $>82 \%$ in all three groups. In the comparison of clinical presentations within the same sex, age rose incrementally from SAP to STEMI for women whereas it fell for men. The proportion of current smokers was highest in STEMI patients for both women and men. Of note, previous PCI was highest in SAP patients for both sexes. The population pyramids of age according to clinical presentation are shown in Figure 2. The differences between women and men were clearly seen, especially for STEMI patients. Within each sex group, $59.6 \%$ were older than 70 years for women compared with $19.9 \%$ for men, among STEMI patients.

Compared to men, women had a lower prevalence of multivessel disease in SAP $(56.2 \%$ vs. $59.2 \%, \mathrm{p}=0.017)$ and a higher prevalence in STEMI ( $60.1 \%$ vs. $51.5 \%$, p < 0.001). Multivessel disease was most frequent in the STEMI group in women, and in the SAP group in men. Left main disease was more common in men compared with women and was seen least frequently in the STEMI group for both sexes. Women had a smaller stent diameter in all three clinical presentations. Stent diameter and stent length were similar irrespective of clinical presentation for women. However, for men, stent diameter was highest and stent length shortest in the STEMI group compared to the other groups. Glycoprotein IIb/IIIa inhibitors were used predominantly in STEMI for both sexes, and among these STEMI patients they were used less in women $(8.0 \%$ vs. $12.0 \%, p=0.009)$. Intravascular ultrasound was used more in men for all clinical presentations, with usage lowest in STEMI for both women and men. Overall first--generation sirolimus-eluting stents were used in $13.1 \%$, whilst new-generation DESs were used in the remaining $87.8 \%$ of the population. The type of stents implanted were similar for all groups (Table 2).

A comparison of the primary endpoint and clinical outcomes at 3 years according to sex and clinical presentation is shown in Table 3 . The rate of the primary endpoint, cardiac death, was highest in the STEMI group for both sexes, with the rate significant higher in women $(7.9 \%$ vs. $4.4 \%, \mathrm{p}=0.001)$. Similarly, all-cause death occurred most frequently in the STEMI group for both sexes, with rates 

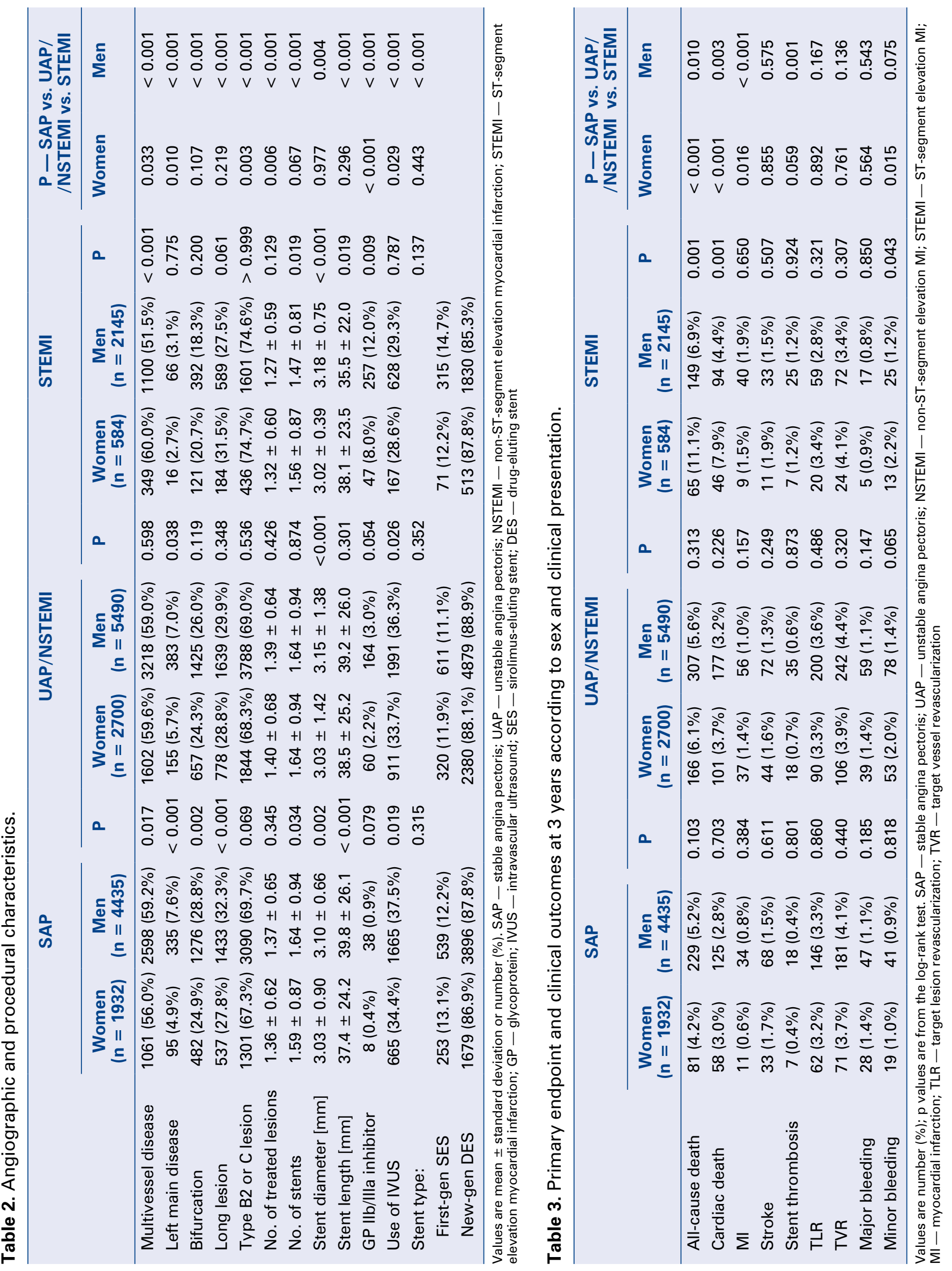


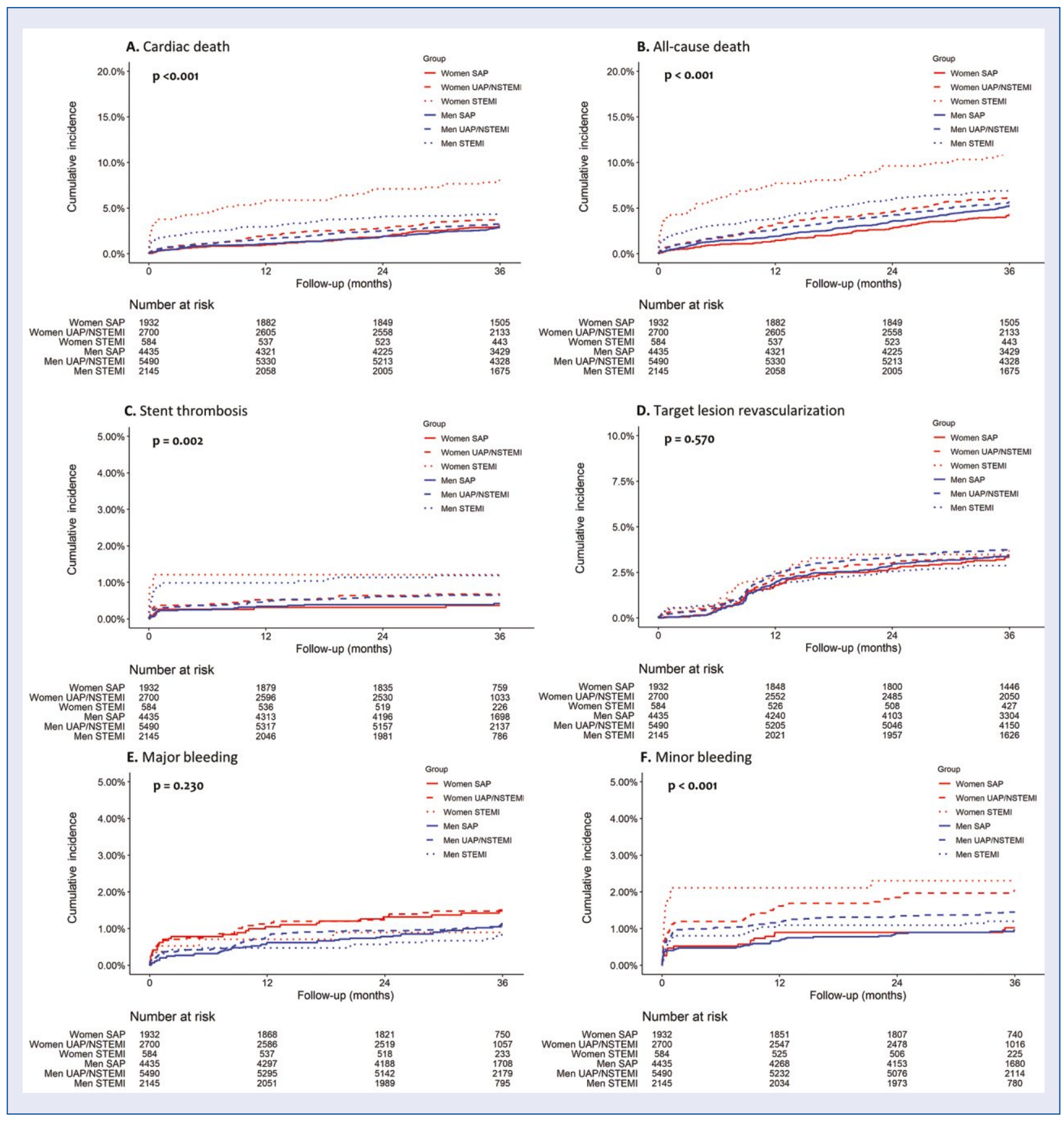

Figure 3. Survival curves according to sex and clinical indication; A. Cardiac death; B. All-cause death; C. Stent thrombosis; D. Target lesion revascularization; E. Major bleeding; F. Minor bleeding; NSTEMI — non-ST-segment elevation myocardial infarction; SAP — stable angina pectoris; STEMI — ST-segment elevation myocardial infarction; UAP - unstable angina pectoris.

higher in women $(11.1 \%$ vs. $6.9 \%, \mathrm{p}=0.001)$. Stent thrombosis was similar between the sexes for all three groups; however, it was highest in the STEMI group among men. Rates of TLR and TVR were similar between women and men, and similar among initial clinical presentations. Although there was no difference in major bleeding according to either sex or clinical presentation; minor bleeding occurred more frequently in female STEMI patients compared with men. Among women, minor bleeding rates were highest in patients with STEMI compared to SAP and UAP/NSTEMI patients.

The Kaplan-Meier survival curves for cardiac death, all-cause death, stent thrombosis, TLR, major bleeding, and minor bleeding are shown in Figure 3. Among the six groups, according to sex 


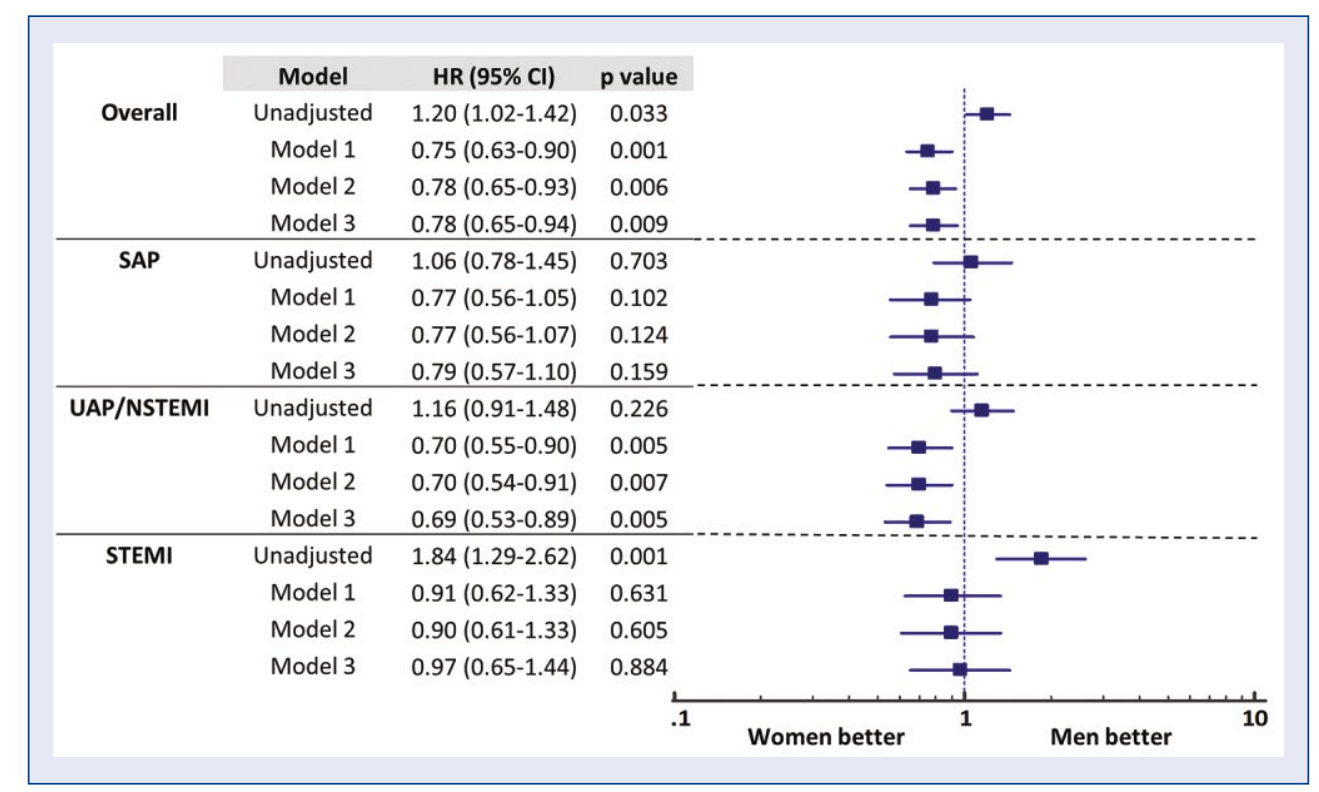

Figure 4. Model comparison of adjusted risk for primary endpoint. The following covariates were sequentially included in the adjusted models: Model 1: age only; Model 2: Model 1 + clinical risk factors; Model 3: Model 2 + angiographic and procedural factors; $\mathrm{Cl}$ - confidence interval; $\mathrm{HR}$ - hazard ratio; NSTEMI — non-ST-segment elevation myocardial infarction; SAP — stable angina pectoris; STEMI — ST-segment elevation myocardial infarction; UAP — unstable angina pectoris.

and clinical presentation, women in the STEMI group had the highest risk of cardiac death, allcause death, and minor bleeding.

To assess the effect of confounders on the primary endpoint, different models were compared in Figure 4. In each clinical presentation, all three models showed similar results, with age the dominant factor in the multivariable analysis. In SAP patients, before and after adjustment, there was no sex difference. In UAP/NSTEMI, the unadjusted results showed no difference between the sexes (hazard ratio [HR] 1.16, 95\% confidence interval [CI] 0.91-1.48, $\mathrm{p}=0.226$ ), but the adjusted results showed a lower risk of the primary endpoint in women (HR 0.69, 95\% CI 0.53-0.89, p = 0.005). In STEMI patients, women had a higher risk of crude outcomes (HR 1.84, 95\% CI 1.29-2.62, $\mathrm{p}=0.001$ ); however, the adjusted results showed a similar degree of risk between women and men (HR 0.97, $95 \%$ CI 0.65-1.44, $\mathrm{p}=0.884$ ). The adjusted results of all outcomes are shown in Figure 5 using model 2. For SAP patients, women had a lower risk of all-cause death (HR 0.57, 95\% CI 0.44-0.74, p < $<0.001)$. For UAP or NSTEMI patients, both allcause death (HR 0.66, 95\% CI 0.54-0.81, $\mathrm{p}<0.001$ ) and cardiac death (HR 0.69, 95\% CI 0.53-0.89, $\mathrm{p}=0.005)$ had a lower risk in women. Note that for STEMI patients, no sex differences were found for any clinical outcomes after adjustment. Table 4 shows the independent predictors for each clinical presentation. Female sex was an independent predictor in UAP/NSTEMI patients but not in SAP and STEMI patients. Age and diabetes were common independent predictors among the three clinical presentation groups.

\section{Discussion}

We investigated the prognostic impact of sex differences in CAD patients after PCI with DES according to clinical presentation. The main findings of the current study are: (1) In the comparison of clinical presentations within the same sex, women presenting with STEMI were older than those with SAP, with the opposite being true among men; (2) In SAP or UAP/NSTEMI patients, there were no differences in crude clinical outcomes between women and men; however, in STEMI patients, women had higher rates of all-cause death, cardiac death, and minor bleeding than men; (3) After multivariable analyses there were similar outcomes between the sexes in STEMI patients.

In our study, women were older than men regardless of clinical presentation, as seen in all previous PCI [8-11] or STEMI studies [12, 13]. In addition, the stepwise increase of age in women, 


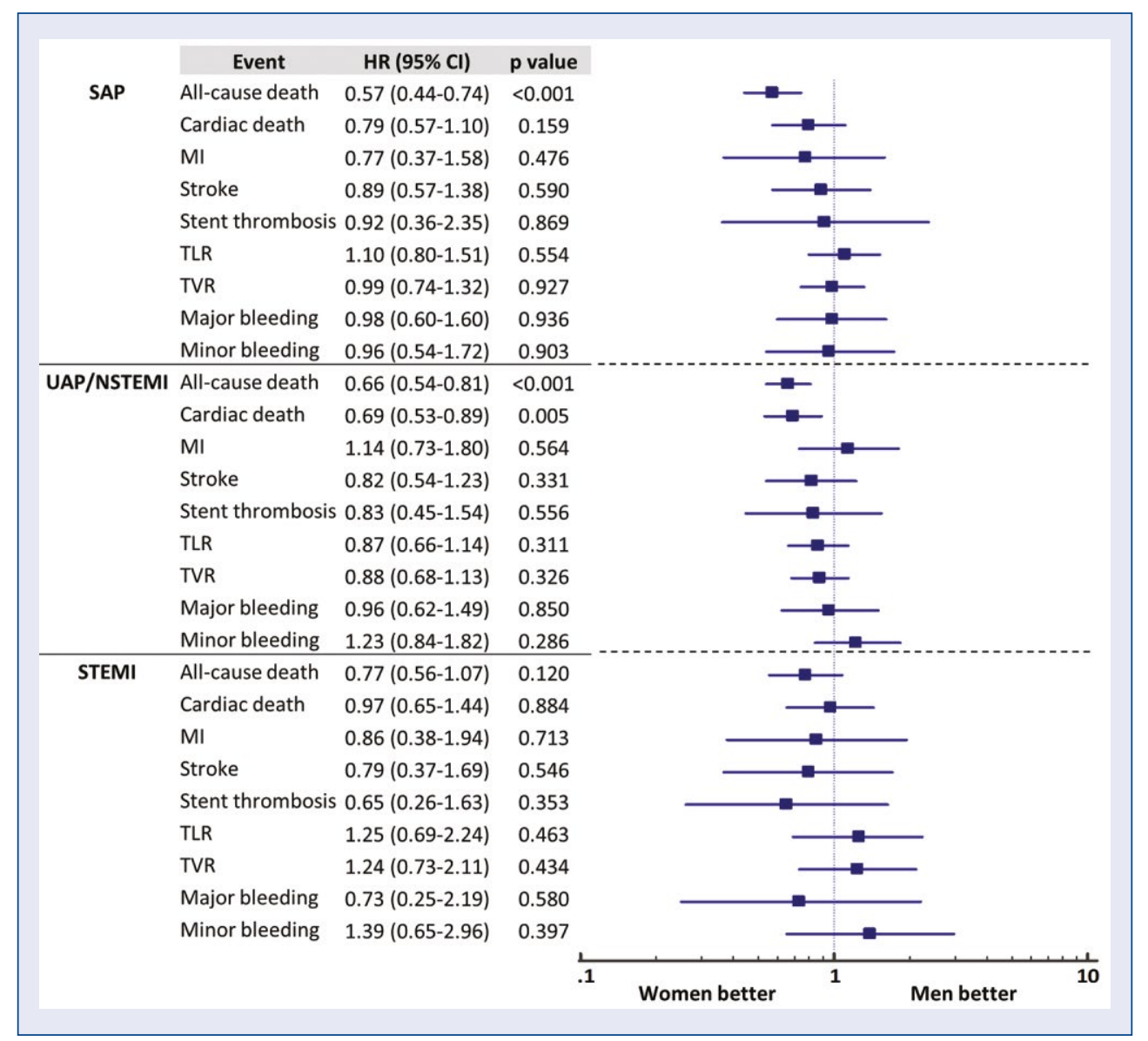

Figure 5. Adjusted risk of women according to clinical presentation; $\mathrm{Cl}$ - confidence interval; $\mathrm{HR}$ - hazard ratio; $\mathrm{MI}$ - myocardial infarction; NSTEMI — non-ST-segment elevation MI; SAP — stable angina pectoris; STEMI — ST-segment elevation MI; TLR — target lesion revascularization; TVR — target vessel revascularization; UAP — unstable angina pectoris.

and the corresponding decrease of age in men, from SAP to STEMI is also similar to previous studies $[14,15]$. The age differences between women and men were 4.7, 6.8, and 11.4 years for SAP, UAP/NSTEMI, and STEMI, respectively. Women diagnosed with STEMI were approximately 10 to 14 years older than men [15-17]. As seen in the model comparison of adjusted risk for the primary endpoint, age played a dominant role in the prognosis of future events. In our previous observational study of plaque characteristics of culprit lesions in STEMI, elderly women had an increased number of vulnerable plaques, implying a lower protective effect of estrogen due to postmenopausal states [16]. A more diffuse pattern of atherosclerosis was observed in women compared to focal lesions in men $[18,19]$. When women get older, a focal obstructive lesion within this diffuse atherosclerosis may result in acute MI. Since plaque erosion is more frequent in women compared with plaque rupture [20], more NSTEMIs may be expected than STEMIs in women compared to men. In our study, the NSTEMI population was made up of $55.4 \%$ women and $44.4 \%$ men. Furthermore, the diffuse progression of plaque in women could explain the older age of female STEMI patients compared to those with SAP. Among STEMI patients, the proportion with age older than 70 years was $59.6 \%$ for women and only $19.9 \%$ for men. These different characteristics of plaque composition and morphology could explain the increased risk of STEMI in women of older age.

There is conflicting evidence regarding the long-term outcomes after PCI between women and men. Previous studies showed better long-term survival either in women or in men compared with the opposite sex [21-25]. In contrast, other studies showed that there is no difference between the sexes, although women had worse baseline characteristics $[2,3,8,10,15]$. Other studies reported 
Table 4. Independent predictors of primary endpoint according to clinical presentation.

\begin{tabular}{|c|c|c|c|}
\hline & $\mathrm{HR}(95 \% \mathrm{CI})$ & Beta coefficient & $\mathbf{P}$ \\
\hline \multicolumn{4}{|l|}{ Overall } \\
\hline Gender (women) & $0.78(0.65-0.94)$ & -0.243 & 0.009 \\
\hline Age & 1.08 (1.07-1.09) & $<0.001$ & 0.080 \\
\hline Previous DM & 1.63 (1.39-1.93) & 0.491 & $<0.001$ \\
\hline Smoking & $1.32(1.08-1.60)$ & 0.277 & 0.006 \\
\hline Previous MI & $1.53(1.14-2.05)$ & 0.422 & 0.005 \\
\hline Previous CHF & $3.22(2.45-4.23)$ & 1.169 & $<0.001$ \\
\hline Previous PVD & $1.74(1.18-2.57)$ & 0.554 & 0.005 \\
\hline Multivessel disease & $1.47(1.21-1.78)$ & 0.382 & $<0.001$ \\
\hline Left main disease & $1.56(1.15-2.11)$ & 0.444 & 0.004 \\
\hline Stent diameter & $0.73(0.59-0.92)$ & -0.308 & 0.007 \\
\hline \multicolumn{4}{|l|}{ SAP } \\
\hline Age & $1.08(1.06-1.10)$ & 0.079 & $<0.001$ \\
\hline Diabetes & 1.48 (1.09-1.99) & 0.390 & 0.011 \\
\hline Previous MI & $1.94(1.16-3.26)$ & 0.664 & 0.012 \\
\hline Previous CHF & $4.52(2.88-7.08)$ & 1.508 & 0.000 \\
\hline Previous PVD & $2.03(1.09-3.76)$ & 0.706 & 0.025 \\
\hline Multivessel disease & $1.46(1.03-2.07)$ & 0.378 & 0.034 \\
\hline \multicolumn{4}{|l|}{ UAP/NSTEMI } \\
\hline Gender (women) & $0.69(0.53-0.89)$ & -0.375 & 0.005 \\
\hline Age & $1.09(1.08-1.11)$ & 0.089 & $<0.001$ \\
\hline Diabetes & $1.88(1.47-2.40)$ & 0.630 & 0.000 \\
\hline Smoking & $1.38(1.03-1.84)$ & 0.319 & 0.032 \\
\hline Previous CHF & $2.86(1.93-4.24)$ & 1.052 & 0.000 \\
\hline Multivessel disease & $1.58(1.16-2.15)$ & 0.457 & 0.004 \\
\hline Bifurcation & $1.37(1.05-1.80)$ & 0.316 & 0.022 \\
\hline Stent diameter & $0.68(0.49-0.95)$ & -0.387 & 0.023 \\
\hline Use of IVUS & $0.72(0.54-0.95)$ & -0.334 & 0.018 \\
\hline \multicolumn{4}{|l|}{ STEMI } \\
\hline Age & 1.07 (1.05-1.08) & 0.064 & $<0.001$ \\
\hline Diabetes & $1.60(1.13-2.28)$ & 0.472 & 0.009 \\
\hline Left main disease & $2.93(1.49-5.77)$ & 1.076 & 0.002 \\
\hline No. of stents & $0.55(0.32-0.94)$ & -0.603 & 0.029 \\
\hline Stent diameter & $0.62(0.38-1.00)$ & -0.482 & 0.048 \\
\hline
\end{tabular}

HR - hazard ratio; CI — confidence interval; SAP — stable angina pectoris; UAP — unstable angina pectoris; NSTEMI - non-ST-segment elevation MI; STEMI - ST-segment elevation MI; MI - myocardial infarction; CHF — congestive heart failure; PVD - peripheral vascular disease; IVUS - intravascular ultrasound

that the greater risk of long-term mortality with women occurred only in STEMI, whilst mortality with other clinical presentations were similar between the sexes $[14,26]$. One of our previous studies on STEMI patients showed that the risk of death and cardiac death in women was significantly higher than in men; however, after adjustment for differences in baseline characteristics, the risk became similar [17].
In the present study, for women, crude cardiac mortality was observed in $3.0 \%$ in SAP, $3.7 \%$ in UAP/NSTEMI, and $7.9 \%$ in STEMI, which is similar to results of a previous study showing $3.5 \%$ in SAP, $3.7 \%$ in UAP/NSTEMI, and 6.4\% in STEMI [26]. In the comparison of women vs. men, SAP and UAP/NSTEMI patients had similar adverse event rates between the sexes, while in STEMI patients, women had a higher risk of all-cause 
death, cardiac death, and minor bleeding. Earlier trials consistently showed a higher incidence of minor bleeding with women than with men, with this effect not seen for major bleeding [27-29]. Our data confirmed this finding. Nevertheless, minor bleeding is of clinical relevance because it leads to medical intervention and influences treatment adherence and health care costs.

Further analysis was conducted to ascertain if there were any confounding factors that could affect clinical outcomes. Our study reported the effect of different adjustment models with age, baseline cardiovascular risk factors, and angiographic/procedural risk factors and showed that outcomes were highly age dependent. Contrary to the unadjusted model, the adjusted results showed lower risk in women compared with men for UAP/ /NSTEMI patients, while there were no sex differences in SAP and STEMI patients. In STEMI patients, women had a higher rate of the primary endpoint than men (HR 1.84, 95\% CI 1.29-2.62, $\mathrm{p}=0.001$ ) at 3 -year follow-up. However, adjusted results showed no difference between the sexes (HR 0.91, 95\% CI 0.62-1.33, p = 0.631). Several long-term outcome studies for STEMI patients also reported that women had an increased risk compared to men, but the difference disappeared after the adjustment for covariates [15, 30-33]. Because the sex differences in long-term outcomes may vary with many confounding factors, the effect of each factor and multifactorial effect should be scrutinized with further research.

Although the GRAND-DES registry enrolled a non-selected population and is thus reflective of real-world cardiovascular care, and multivariable adjustment was performed for significant confounders, it is possible that unmeasured confounders may affect our results. Therefore, we need to interpret them with caution.

\section{Conclusions}

In conclusion, there was no sex difference in cardiac death after PCI with DES for SAP and UAP/ /NSTEMI patients. In STEMI patients, women had worse outcomes compared with men; however, after the adjustment of confounders, female sex was not an independent predictor of mortality.

\section{Funding}

This study was supported by Seoul National University Hospital (SNUH), Republic of Korea (Grant no. 06-2011-3680, 06-2011-3280, 06-2010-1560, 06-2008-2020, 06-2009-2340).
Conflict of interest: Hyo-Soo Kim - institutional research grant from Seoul National University Hospital; the rest of the authors declare no conflict of interest.

\section{References}

1. Kelsey SF, James M, Holubkov AL, et al. Results of percutaneous transluminal coronary angioplasty in women. 1985-1986 National Heart, Lung, and Blood Institute's Coronary Angioplasty Registry. Circulation. 1993; 87(3): 720-727, doi: 10.1161/01. cir.87.3.720, indexed in Pubmed: 8443892.

2. Funakoshi S, Furukawa Y, Ehara N, et al. Clinical characteristics and outcomes of Japanese women undergoing coronary revascularization therapy. Circ J. 2011; 75(6): 1358-1367, doi: 10.1253/ circj.cj-10-0718, indexed in Pubmed: 21483161.

3. Kornowski R, Vaknin-Assa H, Assali A, et al. A comparative analysis of major clinical outcomes with drug-eluting stents versus bare metal stents in male versus female patients. EuroIntervention. 2012; 7(9): 1051-1059, doi: 10.4244/EIJV7I9A167, indexed in Pubmed: 22207229.

4. Park KW, Chae IH, Lim DS, et al. Everolimus-eluting versus sirolimus-eluting stents in patients undergoing percutaneous coronary intervention: the EXCELLENT (Efficacy of Xience/ Promus Versus Cypher to Reduce Late Loss After Stenting) randomized trial. J Am Coll Cardiol. 2011; 58(18): 1844-1854, doi: 10.1016/j.jacc.2011.07.031, indexed in Pubmed: 22018294.

5. Lee JM, Park KW, Han JK, et al. Safety and efficacy of secondgeneration everolimus-eluting Xience $\mathrm{V}$ stents versus zotarolimus-eluting resolute stents in real-world practice: patient-related and stent-related outcomes from the multicenter prospective EXCELLENT and RESOLUTE-Korea registries. J Am Coll Cardiol. 2013; 61(5): 536-544, doi: 10.1016/j.jacc.2012.11.015, indexed in Pubmed: 23273394.

6. Kong MG, Han JK, Kang JH, et al. Clinical outcomes of long stent in the drug-eluting stent era: patient-level pooled analysis from the GRAND-DES registry. EuroIntervention. 2021; 16(16): 1318-1325, doi: 10.4244/EIJ-D-19-00296, indexed in Pubmed: 31543496.

7. Wiviott SD, Braunwald E, McCabe $\mathrm{CH}$, et al. Prasugrel versus clopidogrel in patients with acute coronary syndromes. N Engl J Med. 2007; 357(20): 2001-2015, doi: 10.1056/NEJMoa0706482, indexed in Pubmed: 17982182.

8. Onuma Y, Kukreja N, Daemen J, et al. Impact of sex on 3-year outcome after percutaneous coronary intervention using baremetal and drug-eluting stents in previously untreated coronary artery disease: insights from the RESEARCH (Rapamycin-Eluting Stent Evaluated at Rotterdam Cardiology Hospital) and T-SEARCH (Taxus-Stent Evaluated at Rotterdam Cardiology Hospital) Registries. JACC Cardiovasc Interv. 2009; 2(7): 603-610, doi: 10.1016/j.jcin.2009.03.016, indexed in Pubmed: 19628181.

9. Park DW, Kim YH, Yun SC, et al. Sex difference in clinical outcomes after percutaneous coronary intervention in Korean population. Am Heart J. 2014; 167(5): 743-752, doi: 10.1016/j. ahj.2013.12.027, indexed in Pubmed: 24766986.

10. Josiah A, Farshid A. Gender is not a predictor of mortality or major adverse cardiovascular events in patients undergoing percutaneous coronary intervention for acute coronary syndromes. Heart Lung Circ. 2019; 28(5): 727-734, doi: 10.1016/j. hlc.2018.03.020, indexed in Pubmed: 29705386. 
11. Kim HL, Jang JS, Kim MA, et al. Gender differences of inhospital outcomes in patients undergoing percutaneous coronary intervention in the drug-eluting stent era. Medicine (Baltimore). 2019; 98(20): e15557, doi: 10.1097/MD.0000000000015557, indexed in Pubmed: 31096458.

12. van der Meer MG, Nathoe HM, van der Graaf Y, et al. Worse outcome in women with STEMI: a systematic review of prognostic studies. Eur J Clin Invest. 2015; 45(2): 226-235, doi: 10.1111/ eci.12399, indexed in Pubmed: 25556757.

13. Cenko E, Yoon J, Kedev S, et al. Sex differences in outcomes after STEMI: effect modification by treatment strategy and age. JAMA Intern Med. 2018; 178(5): 632-639, doi: 10.1001/jamainternmed.2018.0514, indexed in Pubmed: 29630703.

14. de Boer SPM, Roos-Hesselink JW, van Leeuwen MAH, et al. Excess mortality in women compared to men after PCI in STEMI: an analysis of 11,931 patients during 2000-2009. Int J Cardiol. 2014; 176(2): 456-463, doi: 10.1016/j.ijcard.2014.07.091, indexed in Pubmed: 25127966.

15. Tang XF, Song Y, Xu JJ, et al. Effect of sex difference in clinical presentation (stable coronary artery disease vs unstable angina pectoris or non-ST-elevation myocardial infarction vs ST--elevation myocardial infarction) on 2-year outcomes in patients undergoing percutaneous coronary intervention. J Interv Cardiol. 2018; 31(1): 5-14, doi: 10.1111/joic.12451, indexed in Pubmed: 29023996.

16. Ann SH, De Jin C, Singh GB, et al. Gender differences in plaque characteristics of culprit lesions in patients with ST elevation myocardial infarction. Heart Vessels. 2016; 31(11): 1767-1775, doi: 10.1007/s00380-016-0806-1, indexed in Pubmed: 26874947.

17. Shin ES, Chung JH, Hahn JY, et al. The clinical impact of sex differences on ischemic postconditioning during primary percutaneous coronary intervention: a POST (the effects of postconditioning on myocardial reperfusion in patients with ST-segment elevation myocardial infarction) substudy. Heart Vessels. 2019; 34(6): 898-905, doi: 10.1007/s00380-018-1316-0, indexed in Pubmed: 30519807.

18. Nasir K, Gopal A, Blankstein R, et al. Noninvasive assessment of gender differences in coronary plaque composition with multidetector computed tomographic angiography. Am J Cardiol. 2010; 105(4): 453-458, doi: 10.1016/j.amjcard.2009.09.053, indexed in Pubmed: 20152238.

19. Smilowitz NR, Sampson BA, Abrecht CR, et al. Women have less severe and extensive coronary atherosclerosis in fatal cases of ischemic heart disease: an autopsy study. Am Heart J. 2011; 161(4): 681-688, doi: 10.1016/j.ahj.2010.12.022, indexed in Pubmed: 21473966.

20. Yahagi K, Davis HR, Arbustini E, et al. Sex differences in coronary artery disease: pathological observations. Atherosclerosis. 2015; 239(1): 260-267, doi: 10.1016/j.atherosclerosis.2015.01.017, indexed in Pubmed: 25634157.

21. Anderson ML, Peterson ED, Brennan JM, et al. Short- and longterm outcomes of coronary stenting in women versus men: results from the National Cardiovascular Data Registry Centers for Medicare \& Medicaid services cohort. Circulation. 2012; 126(18): 2190-2199, doi: 10.1161/CIRCULATIONAHA.112.111369, indexed in Pubmed: 22988009.

22. Bell MR, Grill DE, Garratt KN, et al. Long-term outcome of women compared with men after successful coronary angio- plasty. Circulation. 1995; 91(12): 2876-2881, doi: 10.1161/01. cir.91.12.2876, indexed in Pubmed: 7796495.

23. Gottlieb S, Harpaz D, Shotan A, et al. Sex differences in management and outcome after acute myocardial infarction in the 1990s: A prospective observational community-based study. Israeli Thrombolytic Survey Group. Circulation. 2000; 102(20): 2484-2490, doi: 10.1161/01.cir.102.20.2484, indexed in Pubmed: 11076821.

24. Jacobs AK, Johnston JM, Haviland A, et al. Improved outcomes for women undergoing contemporary percutaneous coronary intervention: a report from the National Heart, Lung, and Blood Institute Dynamic registry. J Am Coll Cardiol. 2002; 39(10): 1608-1614, doi: 10.1016/s0735-1097(02)01835-1, indexed in Pubmed: 12020487.

25. Steg PhG, Greenlaw N, Tardif JC, et al. Women and men with stable coronary artery disease have similar clinical outcomes: insights from the international prospective CLARIFY registry. Eur Heart J. 2012; 33(22): 2831-2840, doi: 10.1093/eurheartj/ ehs289, indexed in Pubmed: 22922505.

26. Giustino G, Baber U, Stefanini GG, et al. Impact of clinical presentation (stable angina pectoris vs unstable angina pectoris or non-ST-elevation myocardial infarction vs ST-elevation myocardial infarction) on long-term outcomes in women undergoing percutaneous coronary intervention with drug-eluting stents. Am J Cardiol. 2015; 116(6): 845-852, doi: 10.1016/j.amjcard.2015.06.010, indexed in Pubmed: 26174605.

27. Czarny MJ, Nathan AS, Yeh RW, et al. Adherence to dual antiplatelet therapy after coronary stenting: a systematic review. Clin Cardiol. 2014; 37(8): 505-513, doi: 10.1002/clc.22289, indexed in Pubmed: 24797884.

28. Straub N, Beivers A, Lenk E, et al. A model-based analysis of the clinical and economic impact of personalising P2Y12-receptor inhibition with platelet function testing in acute coronary syndrome patients. Thromb Haemost. 2014; 111(2): 290-299, doi: 10.1160/TH13-08-0679, indexed in Pubmed: 24154566.

29. Cutlip DE, Kereiakes DJ, Mauri L, et al. Thrombotic complications associated with early and late nonadherence to dual antiplatelet therapy. JACC Cardiovasc Interv. 2015; 8(3): 404-410, doi: 10.1016/j.jcin.2014.10.017, indexed in Pubmed: 25703885.

30. Biava LM, Scacciatella P, Calcagnile C, et al. Sex-related differences in patients with ST-elevation myocardial infarction undergoing primary PCI: A long-term mortality study. Cardiovasc Revasc Med. 2015; 16(3): 135-140, doi: 10.1016/j.carrev.2015.02.001, indexed in Pubmed: 25704157.

31. Hurtado-Martínez J, Pinar-Bermúdez E, Teruel-Carrillo F, et al. [In-hospital and long-term mortality in women with acute myocardial infarction treated by primary angioplasty]. Rev Esp Cardiol. 2006; 59(11): 1113-1122, indexed in Pubmed: 17144986.

32. Jakobsen L, Niemann T, Thorsgaard N, et al. Sex- and age-related differences in clinical outcome after primary percutaneous coronary intervention. EuroIntervention. 2012; 8(8): 904-911, doi: 10.4244/EIJV8I8A139, indexed in Pubmed: 23253544.

33. Wijnbergen I, Tijssen J, van ,t Veer M, et al. Gender differences in long-term outcome after primary percutaneous intervention for ST-segment elevation myocardial infarction. Catheter Cardiovasc Interv. 2013; 82(3): 379-384, doi: 10.1002/ccd.24800, indexed in Pubmed: 23553888. 